\title{
Ein spezielles Avatar-basiertes Beratungsprojekt
}

Um das Beratungsangebot zu vergrößern und um den virtuellen Spielraum für internetbasierte Beratungsangebote zu nutzen, hatte die Hochschule Neubrandenburg eine virtuelle Beratungsambulanz in der anwendererzeugten virtuellen 3-D-Welt des Internets, auf der Plattform Second Life (SL; vgl. http://secondlife. com) getestet (Bräutigam et al. 2011).

Um Erkenntnisse über die Nutzerakzeptanz gegenüber virtuellen Beratungsangeboten zu bekommen, wurde eine Vorabstudie zu den Ansprüchen und der Kritik der User an bisherigen Angeboten angestellt. Diese ersten Ergebnisse mündeten in der Fragestellung, wie Avatar-basierte Beratungsangebote in SL wahrgenommen werden. Aus den Resultaten lassen sich ähnliche Ansprüche wie bei Beratungsangeboten des Real Life (RL) vermuten (Lüngen und Rienitz 2010). Es wurde deutlich, dass den Nutzern ein formal korrektes, transparentes und am realen Leben (RL) überprüfbares Auftreten der Forscher und Berater sehr wichtig ist. Sie legten Wert auf die Identifizierbarkeit des Ansprechpartners und auf seine nachweislichen Qualifikationen. Weiterhin erwarteten die User, dass der Forscher ein angemessenes Interesse an SL hat, über einen SL-Account verfügt und über längere Zeit inworld präsent war. Neben Offenheit und Wertschätzung aufseiten des Beraters (und Forschers) wird von den Usern ein besonderer Wert auf den sensiblen Umgang mit Daten gelegt. „Als Voraussetzung für die Gewährleistung eines produktiven Kontaktes sehen wir das Verständnis für die (negativen) Erfahrungen der User und empfehlen diese zu beachten" (Lüngen und Rienitz 2010, S. 28).

Aus dieser Vorstudie wird ersichtlich, dass man von einer anspruchsvollen potenziellen Nutzergruppe der virtuellen Beratungsangebote ausgehen darf und es Sinn macht, das neue Format des Beratungsangebotes einmal genauer in den Blick zu nehmen und nicht zuletzt Unterschiede zum realen Beratungssetting aufzuzeigen. 